\begin{tabular}{|c|c|c|c|}
\hline Eiszeitalter u. Gegenwart & 49 & $\begin{array}{c}132-143 \\
4 \mathrm{Abb} .\end{array}$ & Hannover 1999 \\
\hline
\end{tabular}

\title{
Pollenstratigraphische Gliederung des Spätglazials im Rheinland
}

\author{
URSULA SCHIRMER*) \\ - Meiendorf Interstadial, boundary Pleniglacial/Lateglacial, Schleswig-Holstein, Eifel, Lahn, \\ Weser, Central Europe -
}

\begin{abstract}
Kurzfassung: Die vorliegende Arbeit befaßt sich mit dem Meiendorf-Interstadial nach der Definition von Menke (1968). Das Meiendorf-Interstadial ist eine vorböllingzeitliche Wärmeschwankung, die durch einen Anstieg von Zwergbirke (Betula nana), Sanddorn (Hippophä̈) und Beifuß (Artemisia) charakterisiert ist. Es wurde erstmals 1968 von MENKE für Schleswig-Holstein vorgestellt und konnte seitdem im Rheinland (SCHIRMER 1995, 1996; LiTT \& STEBiCH 1996), an der Lahn (SCHIRMER 1998) und an der Weser (MerkT \& Müller im Druck) nachgewiesen werden. Sowohl aus den östlichen Niederlanden als auch aus dem mitteldeutschen Raum und dem Alpenvorland sind Pollenprofile bekannt, in denen sich Hinweise auf diese vorböllingzeitliche Wärmeschwankung finden. Daher kann man von einem mitteleuropäischen Geltungsbereich für das MeiendorfInterstadial ausgehen. Das Meiendorf-Interstadial erscheint demnach geeignet, eine einheitliche Abgrenzung vom Hochglazial zum Spätglazial zu erbringen. Es gibt zahlreiche Hinweise auf weitere mögliche Wärmeschwankungen, die jünger als das letztglaziale Maximum (LGM) und älter als das Bölling-Interstadial sind. Aufgrund der Tatsache, daß es sich hierbei um schwimmende Interstadiale handelt, denen der palynologische Anschluß an das bekannte Spätglazial fehlt und deren zeitliche Stellung oft nur durch ${ }^{14} \mathrm{C}$-Daten belegt wird, ist eine Korrelation mit dem Meiendorf-Interstadial bisher nicht möglich.
\end{abstract}

\section{[Pollenstratigraphical subdivision of the Lateglacial in the Rheinland]}

Abstract: The present text is concerned with the Mei-
endorf Interstadial which has been established by MEN-
KE 1968 on pollen diagrams of Schleswig-Holstein. It is
older than the Bölling Interstadial and is characterized
by a rise of Betula nana, Hippophaë and Artemisia pol-
len. Meanwhile its validity has been proved on pollen
profiles of the Eifel (SCHIRMER 1995, 1996; LITT \& STEBICH
1996), the Lahn river (SCHIRMER 1998) and the Weser ri-
ver (MERKT \& MüLLe in print). Some pollen records of
the eastern Netherlands, the central part of Germany
and the Alpine foreland with a long Lateglacial sequence
provide hints on the existence of a climatic amelio-

*) Anschrift der Verfasserin: Dr. U. Schirmer, Abt. Geologie der Heinrich-Heine-Universität, Universitätsstr. 1, D-40225 Düsseldorf ration older than the Bölling Interstadial. Thus the Meiendorf Interstadial proves to be a reliable event in central Europe. It provides a useful indicator for a biostratigraphic boundary between the Pleniglacial and Lateglacial period. There exist numerous evidence for interstadial phases between the Last Glacial Maximum (LGM) and the Bölling Interstadial. They are reported from the northern and the southern hemisphere. Their stratigraphical position in general is given by ${ }^{14} \mathrm{C}$-dating, and their geological position lies between the moraines of the LGM and phases of glacier retreat. Whenever they provide palynological data, the record is a floating one that lacks the direct connection with the palynologically known Lateglacial. For the time being it is not recommended to correlate them with the Meiendorf Interstadial.

\section{Das Meiendorf-Interstadial im norddeutschen Spätglazial}

Bei den Ausführungen zum Spätglazial werden folgende biostratigraphische Einheiten benutzt:

Dryas 3

Alleröd-Interstadial

Dryas 2

Bölling-Interstadial

Dryas 1

oder Jüngere Tundrenzeit

Meiendorf-Interstadial

Hochglazial

(Dryas 1, 2 und 3 nach Nilsson 1961 und Ältere, Mittlere und Jüngere Tundrenzeit sensu MEnkE (in Bock et al. 1985)

1968 stellte Menke im Profil Glüsing (West-Schleswig-Holstein; Abb.1) das „Meiendorf-Intervall“ vor und wies es auch im Profil Dätgen, das von Aletsee 1959 bearbeitet worden war, nach. 1985 bezeichnete es Menke in Bock et al. (1985) endgültig als Meiendorf-Interstadial und schlug es in den 90er Jahren der Stratigraphischen Kommission als Holostratotypusprofil des Meiendorf-Interstadials für Nordwestdeutschland vor. Der Name Meiendorf ist einer jungpaläolithischen Rentierjägerfundstelle im Nordosten von Hamburg entnommen, die unter anderem eng mit den Arbeiten von SchÜtrumpF $(1936,1955)$ verbunden ist. 


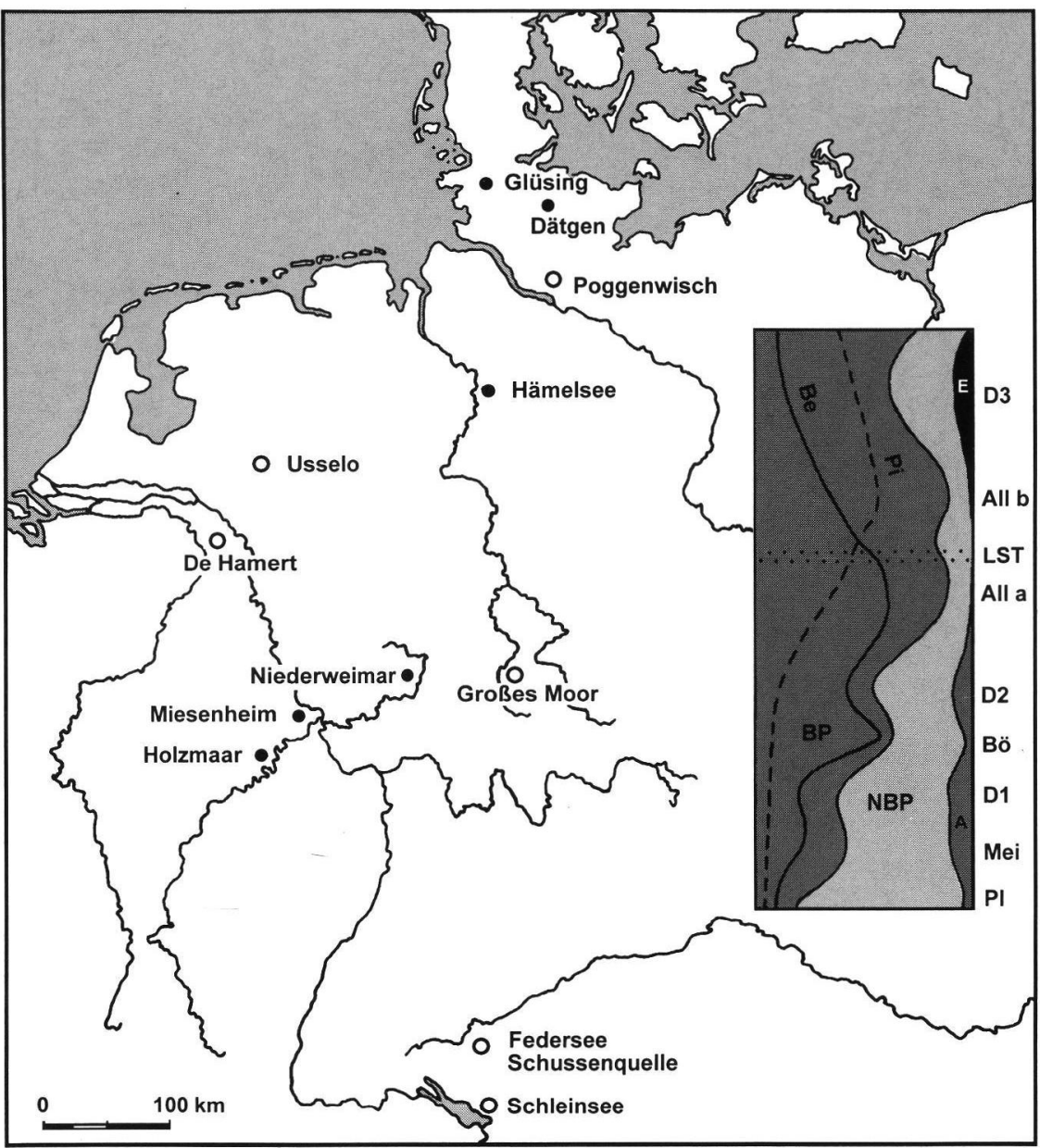

Abb. 1: Lageplan der im Text genannten Lokalitäten von Pollenprofilen. Schwarze Punkte: Pollenprofile mit Meiendorf-Interstadial; offene Kreise: Profile, die Hinweise auf das Meiendorf-Interstadial enthalten $; \mathrm{Pi}=$ Pinus, $\mathrm{Be}=$ Betula, $\mathrm{A}=$ Artemisia, $\mathrm{E}=$ Empetrum, $\mathrm{NBP}=$ Nichtbaumpollen, $\mathrm{BP}=\mathrm{Baum}-$ pollen, LST $=$ Laacher See-Tephra

Fig. 1: Location map of all mentioned pollen profiles. Filled circles: pollen records which include the Meiendorf Interstadial; open circles: profiles with suggested evidences for the Meiendorf Interstadial; Pi $=$ Pinus, $\mathrm{Be}=$ Betula, $\mathrm{A}=$ Artemisia, $\mathrm{E}=$ Empetrum, $\mathrm{NBP}=$ nonarboreal pollen, $\mathrm{BP}=$ arboreal pollen, $\mathrm{LST}=$ Laacher See Tephra

Menke legte im Profil Glüsing die Grenze zwischen Hochglazial und Meiendorf an den Anstieg der Artemisia-Kurve. Erhöhte Werte des Pollens von Zwergbirke (Betula nana) und Beifuß (Artemisia) sowie das gleichzeitige oder unmittelbar nachfolgende Maximum von Sanddorn (Hippophaё) kennzeichnen das Meiendorf-Interstadial selbst. Die Abgrenzung des Meiendorf-Interstadials nach oben erfolgt durch einen Rückgang der Birkenwerte und einen Nichtbaumpollenanstieg unter hoher Beteiligung von Helianthemum und Artemisia, die die Dryas 1 charakterisieren.
Demzufolge handelt es sich bei der pollenstratigraphischen Charakteristik des Meiendorf-Interstadials um den ersten von insgesamt drei Gipfeln der Betula-Kurve, der hier überwiegend durch Zwergbirke verursacht wird. Ein Erkennungsmerkmal für diesen ersten Birkengipfel ist auch, daß er das Hippophaë-Maximum im Gefolge hat. Der zweite Betula-Gipfel liegt im Bölling, wenn die Hippophaë-Werte bereits stark zurückgegangen sind. Der dritte Birkengipfel fällt ins frühe Alleröd, wo der NBP seinen spätglazialen Tiefststand erreicht. 
Usinger (1985) gliedert anhand eigener Pollenprofile in Schleswig-Holstein das Spätglazial in pollen assemblage zones (paz), um frei von stratigraphischen Zuordnungen die Vegetationsentwicklung darzustellen. Damit wollte Usinger den Problemen der Parallelisierung der als böllingzeitlich eingestuften Pollendiagramme in Norddeutschland und angrenzenden Gebieten auf den Grund gehen, die vermutlich durch die Verknüpfung unterschiedlich alter Diagrammabschnitte verursacht werden. Wie oben dargelegt gibt es im Spätglazial Nord- und Westdeutschlands drei $B e-$ tula-Gipfel, so daß der Fall eintreten könnte, daß man irrtümlich den ersten mit dem zweiten oder den zweiten mit dem dritten verknüpft, wenn nur zwei Gipfel vorhanden sind und die Begleitmerkmale nicht berücksichtigt werden können. Das würde erklären, warum verschiedene böllingzeitlich eingestufte Pollendiagramme oft einen von einander abweichenden Entwicklungsstand der Vegetation präsentieren, obwohl sie nicht aus unterschiedlichen Klimabereichen stammen. In UsingERs paz-Gliederung des Spätglazials schließt sich einer Zone, die mit ansteigenden ArtemisiaWerten den Übergang zwischen hochglazialen und spätglazialen Verhältnissen darstellt, die klimatisch günstigere Hippophaë-Betula nana-paz an. Sie zeichnet sich durch Betula nana-Werte bis 35\% und maximale Hippophaë- und JuniperusWerte aus. Damit entspricht sie nach pollenanalytischen und stratigraphischen Merkmalen dem Meiendorf-Interstadial sensu Menke.

Mit der Ausgliederung des Meiendorf-Interstadials beginnt das Spätglazial mit einem, wenn auch schwachen Interstadial. Vorher war eine lange kontroverse Diskussion darüber geführt worden, wann der Übergang des vegetationskargen Hochglazials zur Tundrenvegetation des Spätglazials zu ziehen sei (vgl. Firbas 1949: 48, vaN DER HAMMEN 1951). Innerhalb der ersten Zeit vorsichtiger Vegetationsentfaltung, Tundren- oder Dryaszeit genannt, wurden dann Erwärmungsphasen wie das Alleröd- (Hartz \& Milthers 1901) und das Bölling-Interstadial (IVERSEN 1942) ausgeschieden. Dadurch erhielt der Begriff Tundren- oder Dryaszeit, der gegenüber dem Hochglazial eine klimatische Gunstzeit darstellte, die Bedeutung eines Klimarückschlages zwischen den wärmeren Interstadialen. Die gegensätzliche Anwendung des Begriffs Tundren- oder Dryaszeit ist also forschungsgeschichtlich begründet:

Vor IVERSEN (1942) gliederte man das Spätglazial dreiteilig in Ältere Dryaszeit (I), Alleröd (II) und Jüngere Dryaszeit (III) (vgl. SchütrumpF 1955). In dieser Gliederung ging das Meiendorf-Interstadial in der Älteren Dryas (I) auf. Als IvERSEN (1942) mit dem Bölling-Interstadial (Ib) eine präallerödzeitliche klimatische Gunstphase aufstellt, wird das Spätglazial fünfteilig. Die Ältere Dryas (I) wird jetzt in die vorböllingzeitliche Älteste Dryas (Ia) und die nachböllingzeitliche Ältere Dryas (Ic) aufgeteilt. Das Meiendorf-Interstadial ging nun folglich in der Ältesten Dryas (Ia) auf. Damit wich der umfassendere Begriff Ältere Dryas (I) einer Aufspaltung in Älteste (Ia) und Ältere (Ic) Dryas. NiLssON (1961) vereinfacht die nun sprachlich kompliziert gewordene Nomenklatur der Tundren- oder Dryaszeiten durch eine Numerierung in Dryas 1-3 von alt nach jung.

Bis zur Etablierung des Meiendorf-Interstadials als ersten pollenanalytischen Abschnitt nach dem Hochglazial begann das Spätglazial nomenklatorisch mit einer Tundrenzeit. Diese Tundrenzeit, sei es die Ältere, Älteste oder Dryas 1, war zum Hochglazial im Liegenden hin nicht begrenzt. Mit der Abtrennung der basalen leichten Erwärmung des Meiendorf-Interstadials beginnt das Spätglazial mit einem schwachen Interstadial und die erste Dryas- oder Tundrenzeit liegt zwischen Meiendorf- und Bölling-Interstadial. Damit erfährt das Spätglazial eine klare Abgrenzung zum liegenden Hochglazial.

\section{Die Spätglazialgliederung Nord- und Westdeutschlands}

Der Verlauf des Spätglazials in Nordwestdeutschland ist im Inset der Abb. 1 schematisch dargestellt: Nach dem Meiendorf-Interstadial und der nachfolgenden Dryas 1 ist das Bölling durch den kräftigen Anstieg der Baumbirken auf über $60 \%$ und den korrespondierenden NBP-Rückgang charakterisiert. In der nachfolgenden Klimaverschlechterung der Dryas 2 steigen die Werte des Nichtbaumpollens, besonders von Artemisia erneut. Das Alleröd-Interstadial kann in zwei, mancherorts auch in drei Abschnitte unterteilt werden: Im Falle der Zweiteilung dominiert im älteren Alleröd Betula, im jüngeren Alleröd nehmen die Pinus-Werte zu. Dort wo die Laacher See-Tephra zur Ablagerung gekommen ist, besitzen die Pollenprofile eine wertvolle Zeitmarke. Die Überkreuzung der Betula- durch die Pinus-Kurve liegt meistens in Nähe dieses Tephrahorizonts. Im Nordwesten Mitteleuropas allerdings bleibt in den meisten Fällen Betula während des gesamten Alleröds dominant. Im Falle einer Dreiteilung zeichnet sich im mittleren Alleröd zusätzlich eine 
Phase erhöhter NBP-Werte ab; zum Beispiel im Profil De Hamert (Teunissen 1983). Sie liegt in De Hamert im von Betula dominierten Profilteil. Die Jüngere Tundrenzeit präsentiert einen deutlichen NBP-Gipfel, in Nordwestdeutschland unter hoher Beteiligung von Empetrum.

Seit vielen Jahren sind die palynologischen Ergebnisse vom Hämelsee bekanntgemacht worden (MerkT \& Müller im Druck). Im MeiendorfInterstadial folgt hier auf den ersten Betula- und Artemisia-Anstieg unmittelbar das HippophaëMaximum. So dient das Pollenprofil Hämelsee als Brückenpfeiler zwischen Norddeutschland und dem Rheinland (s. Abb.1).

\section{Pollenprofile mit Meiendorf-Interstadial im Rheinland}

1995 wurde das Profil Miesenheim (Abb. 2 u. 3) auf dem 14. INQUA-Kongreß zum ersten Mal vorgeführt und im Exkursionsführer veröffentlicht (SCHIRMER 1995). Es stammt aus einem verlandeten See, in dem aus einem Profilquerschnitt die einzige vorhandene ungestörte Stelle beprobt wurde. Das Profil zeigt lithologisch den Übergang von hochglazialem Löß zu einer Wechsellagerung von Torf und Kalkmudde. In den Werten der organischen Substanz (Abb. 3) spiegelt sich der Übergang vom hochglazialen Löß zur spätglazialen Mudde-Torf-Wechsellage sowie das Einsetzen des Allerödtorfes wider. Das Meiendorf-Interstadial grenzt sich deutlich vom hochglazialen Löß ab: Während im pollenarmen Löß ferntransportierter Pinus-Pollen neben geringen Betula- und NBP-Vorkommen das Pollenspektrum dominiert, steigen zu Beginn der spätglazialen Pollenspektren besonders die Betula-, Artemisia- und Gramineen-Werte deutlich an. Hier kennzeichnen die spätglazialen Pioniere und Heliophyten, besonders Juniperus, Ephedra und Salix, Helianthemum, Potentilla, Thalictrum und Filipendula, das Pollenspektrum des Meiendorf-Interstadials. Die folgende Dryas 1 ist durch das absolute Artemisia-Maximum belegt. In diesem Bereich liegt auch der Gipfel der Hippophaë-Kurve. Das Bölling ist durch das scharf abgegrenzte absolute $B e$ tula-Maximum und einen NBP-Tiefststand ausgewiesen. Der Bereich der Pollenzone D 2 zwischen Bölling und Alleröd tritt im Diagramm durch Pollenspektren mit einem sekundären ArtemisiaGipfel hervor. Es schließt sich die Betula-Phase des Alleröds an, zu deren Ende sich die Kurven von Pinus und Betula annähern. Die Kreuzung ihrer Kurven liegt deutlich unterhalb des Horizonts der Laacher See-Tephra, die unmittelbar über der obersten Pollenprobe das Profil plombiert. Die Pinus-Phase des Alleröds mit anhaltend hohen Filipendula-Werten ist im Profil Miesenheim noch mit $6 \mathrm{~cm}$ Mächtigkeit erhalten.

1996 haben LITT \& STEBICH in den weiter westlich gelegenen laminierten Seeablagerungen des Profils Holzmaar ebenfalls das Meiendorf-Interstadial ausgeschieden. Das Meiendorf-Interstadial im Profil Holzmaar ist wie auch im Profil Miesenheim durch einen deutlichen Anstieg von Betula und Artemisia gekennzeichnet. Die Spektrenfolge zwischen dem Meiendorf- und dem Bölling-Interstadial (D $1 \mathrm{im}$ Profil Meiendorf) wird dort noch einmal durch einen eingeschalteten ,Betula-Gipfel unterteilt.

Das Pollenprofil Niederweimar (Abb. 4) (SCHIRMER 1998) liegt im Bereich der hochwürmzeitlichen Niederterrasse der Lahn. Es beginnt direkt über dem Terrassenschotter. Der hochglaziale Schotter ist pollenfrei. Die Auensedimente beginnen mit dem ausklingenden Meiendorf-Interstadial. Dieses ist an der Basis des Pollenprofils noch eindeutig erfaßt: mit Betula-Werten von über $30 \%$, Artemisia-Werten zwischen 20 und 30\%, sowie Vorkommen von Hippophaë, Juniperus und Helianthemum. Die meiendorfzeitlichen hohen Betula-Werte gehen zur Dryas 1 deutlich zurück. Wie in Miesenheim und im Holzmaar liegt in der Dryas 1 das absolute Maximum von Artemisia, hier mit der Besonderheit, daß die Prozente doppelt so hoch wie bei den westlicheren Profilen sind, wohl durch die Lage im Niederschlagsschatten des Rheinischen Schiefergebirges bedingt. Auch hier liegt das absolute Betula-Maximum im Bölling, auch hier zeigt die Dryas 2 ein sekundäres Maximum von Artemisia sowie einen deutlichen NBP-Gipfel. Das Alleröd ist durch eine $B e-$ tula-Phase und eine Zeit der wiederholten Kreu-

Legende zur Lithologie der Pollenprofile in den Abb. 2, 3, 4.

Lithological symbols for the pollen profiles in the Fig. 2, 3, 4 .
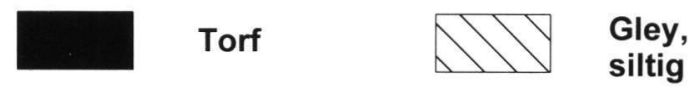

\section{川D $\begin{aligned} & \text { Mudde, } \\ & \text { torfstreifig }\end{aligned} \therefore . . \quad \begin{aligned} & \text { Gley, } \\ & \text { sandig }\end{aligned}$}

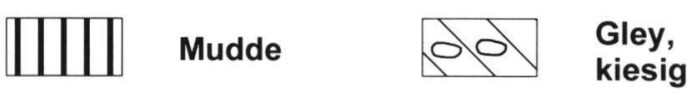




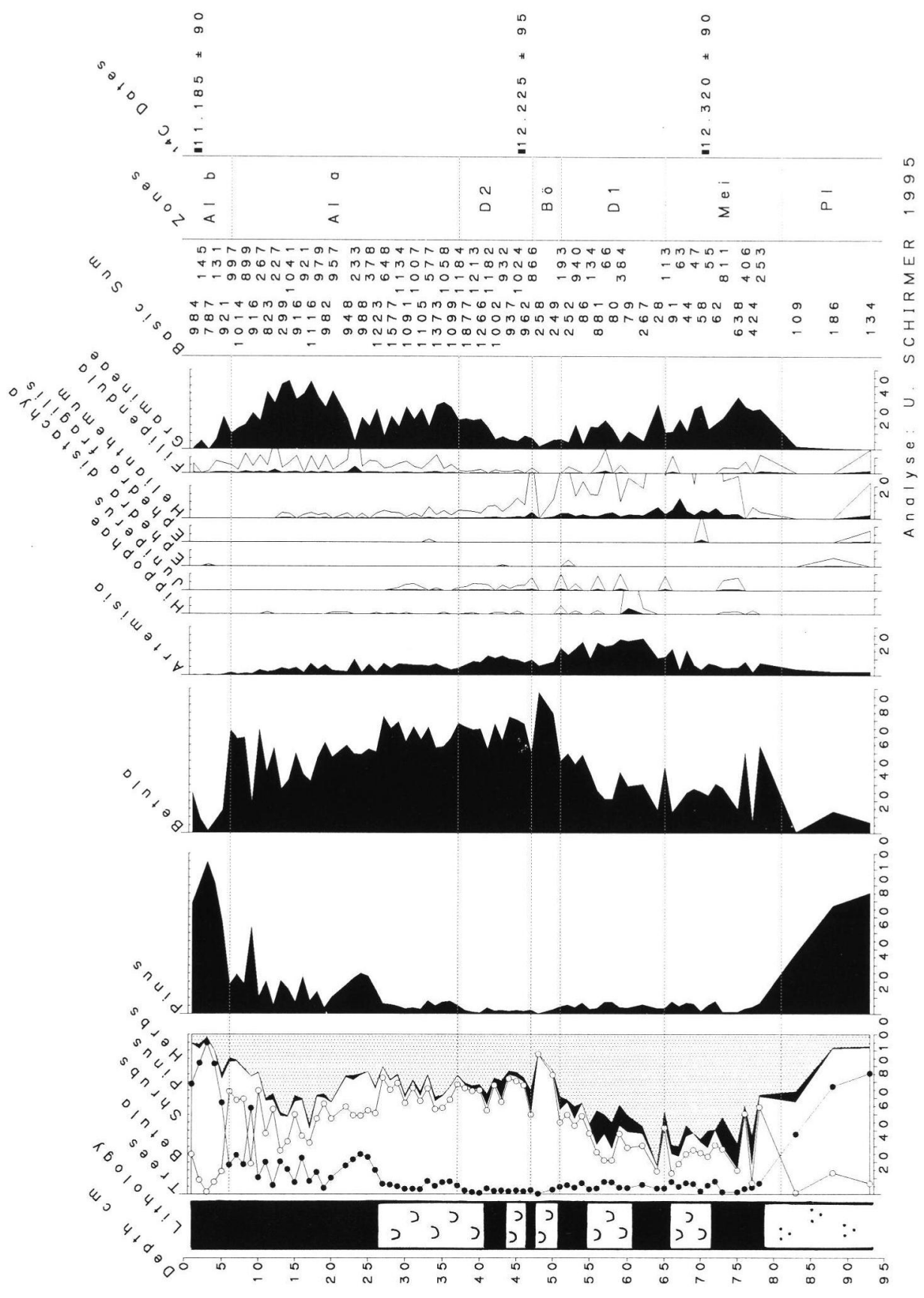

Abb. 2: Pollenprofil Miesenheim mit ausgewählten Taxa, Überhöhung 10-fach, Legende zur Lithologie siehe Abb. 5. Über dem obersten Pollenspektrum begrenzt die Laacher See-Tephra das Pollenprofil.

Fig. 2: Pollen profile Miesenheim with selected taxa, exaggeration factor 10, lithological symbols see Fig. 5 , Laacher See Tephra lies immediately on top of the uppermost pollensample

Abb. 3 (siehe Seite 137): Pollenprofil Miesenheim, vollständiges Diagramm, Überhöhung 5-fach (SCHIRMER 1996), Legende zur Lithologie siehe Abb. 5. Über dem obersten Pollenspektrum begrenzt die Laacher See- Tephra das Pollenprofil

Fig. 3: Pollen profile Miesenheim, complete diagram, exaggeration factor 5 (Schirmer 1996), Laacher See

Tephra lies immediately on top of the uppermost pollensample 


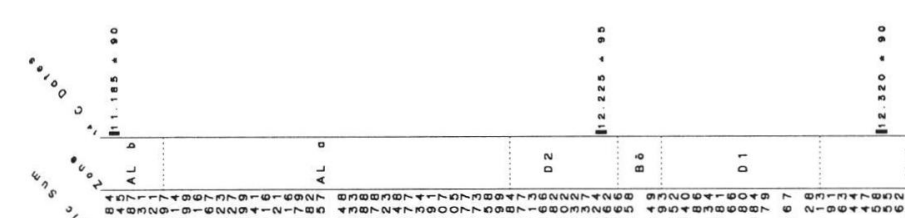

$:$
$\vdots$
$\vdots$
0
0

$=$

$\frac{1}{2}$

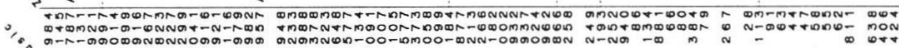

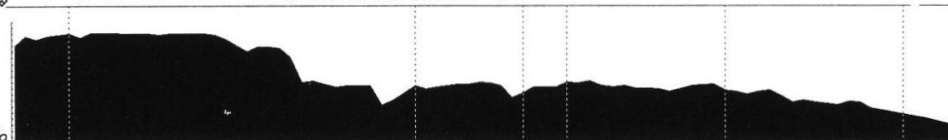

"



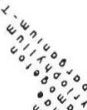

$\therefore$ ".



m N



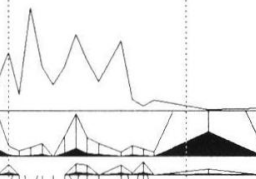

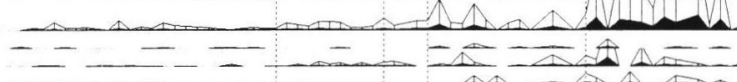

$\longrightarrow$

$\Delta \Delta-Z \Delta \Delta$

$\Delta \Delta$



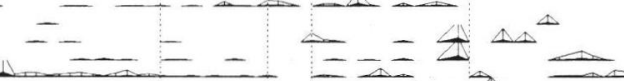

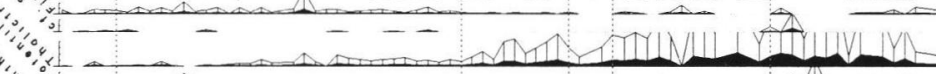

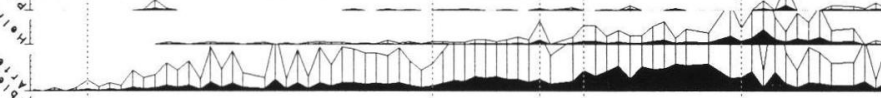

$\therefore 1$

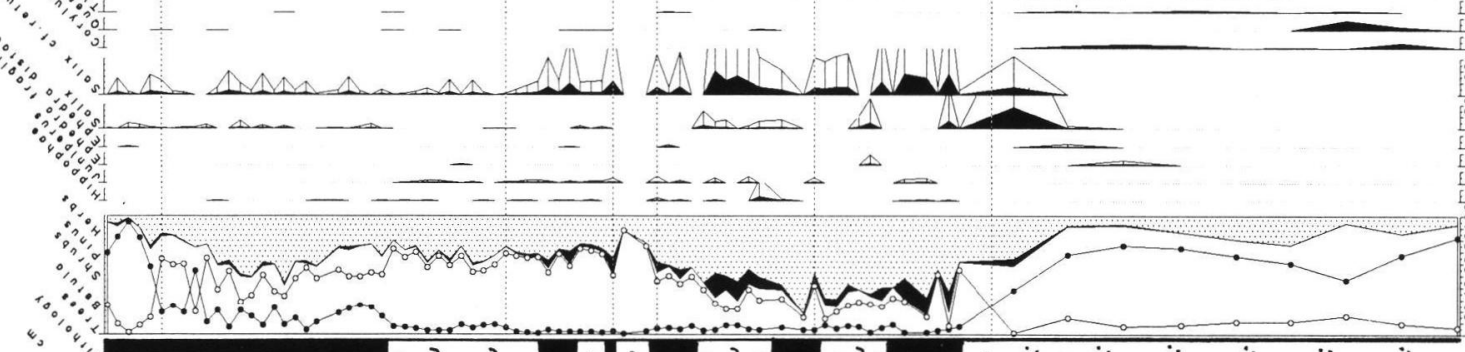
$32,5,5] 5]$ 


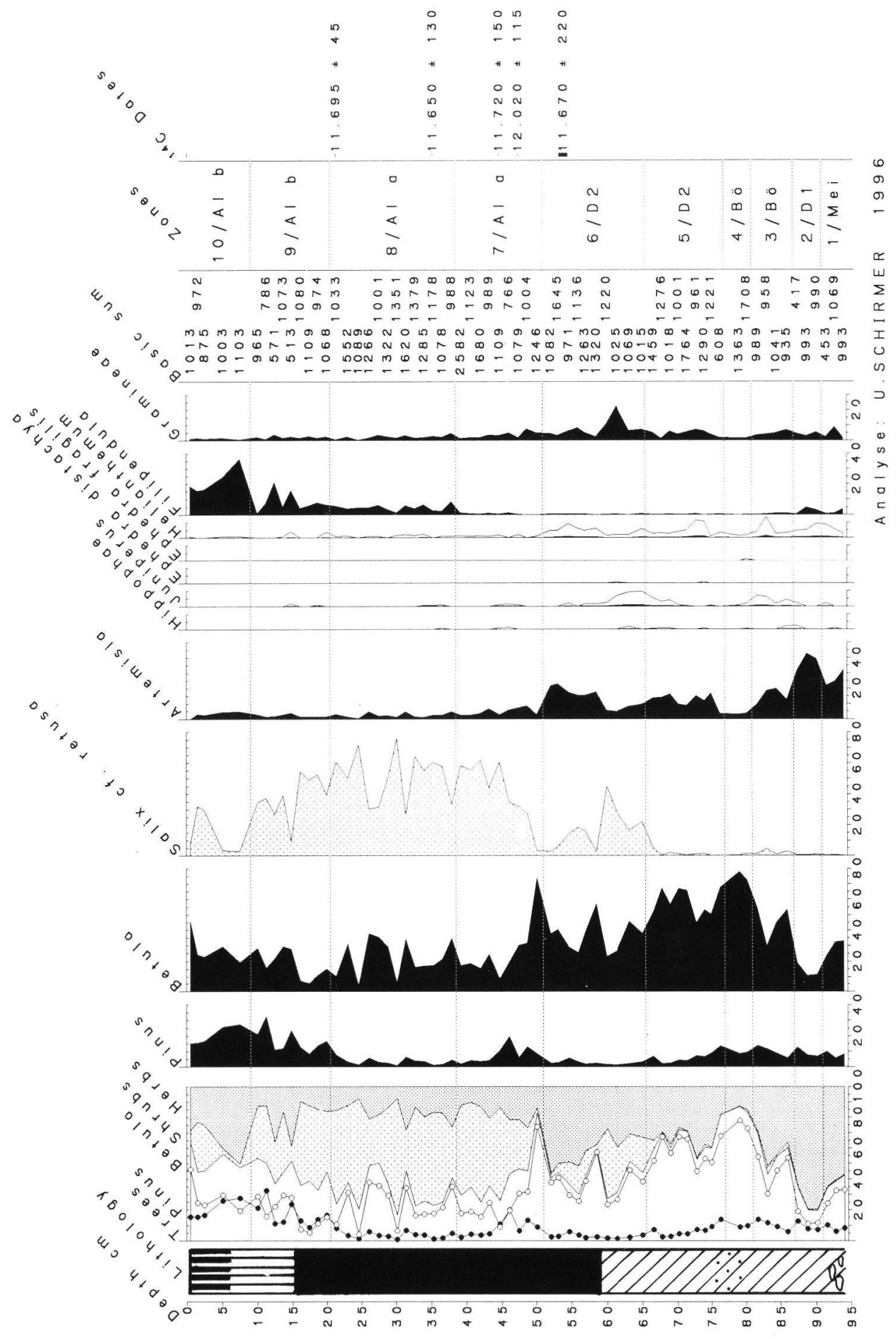

Abb. 4: Pollenprofil Niederweimar mit ausgewählten Taxa, Überhöhung 10-fach, Legende zur Lithologie siehe Abb. 5. Über dem obersten Pollenspektrum begrenzt die Laacher See-Tephra das Pollenprofil

Fig. 4: Pollen profile Niederweimar with selected taxa, exaggeration factor 10, lithology symbols see Fig. 5. Laacher See Tephra lies immediately on top of the uppermost pollensample 
zungen der Pinus- und Betula-Kurve gekennzeichnet. Auch hier befindet sich im Top des Profils unmittelbar über der höchsten Pollenprobe die Laacher See-Tephra als Zeitmarke. Wie dargestellt läßt sich das Pollenprofil Niederweimar also bestens mit den Profilen Miesenheim und Holzmaar parallelisieren.

An dieser Stelle wird bewußt auf die Diskussion der Radiokarbondaten für das Spätglazial verzichtet. Meine zur Einstufung herangezogenen Argumente bauen rein auf der Palynostratigraphie auf. Die pollenstratigraphischen Zonen Meiendorf, Dryas 1, Bölling und Dryas 2 sind von so kurzer Dauer, daß sie häufig innerhalb der bei der ${ }^{14} \mathrm{C}$ Datierung angegebenen Fehlergrenzen liegen. Berücksichtigt man nun noch die im Spätglazial vorhandenen bekannten Plateaus, die sich aus dem Vergleich von ${ }^{14} \mathrm{C}$-Daten und Kalenderjahren ergeben, so bleibt eine Zonierung dieses frühen Spätglazials nach Radiokarbonjahren spekulativ. Dennoch fügt sich das in Abbildung 2 und 3 des Pollenprofils Miesenheim angegebene ${ }^{14} \mathrm{C}$-Alter an Holz für das Meiendorf-Interstadial von $12.320 \pm 90$ a $\mathrm{BP}$ in den von MeNke für das Meiendorf gesteckten Zeitraum (vgl. Kapitel 5).

\section{Hinweise auf die Existenz des Meiendorf- Interstadials im weiteren Mitteleuropa}

Bis in die 90er Jahre wurde die Abgrenzung des Meiendorf-Interstadials nur in Norddeutschland vorgenommen. Die dargestellten Ergebnisse belegen die problemlose Anwendbarkeit der Spätglazialgliederung sensu MENKE via Hämelsee (MERKT \& Müller im Druck) nach Süden bis ins Rheinland (Miesenheim: SCHIRMER 1995, 1996; Holzmaar: LitT \& SteBich 1996 und Niederweimar: SCHIRMER 1998) (Abb. 1).

Außerhalb dieses Raumes ist in den Pollenprofilen als älteste klimatische Gunstphase das Bölling-Interstadial anzutreffen. Hierbei erheben sich die Fragen, ob in diesen Profilen die Sedimente der biostratigraphischen Meiendorf-Periode nicht zur Ablagerung gelangt sind, eventuell wieder abgetragen wurden oder in den als böllingzeitlich eingestuften Profilabschnitten enthalten sind. In Abbildung 1 sind daher zusätzlich die Standorte einiger Pollenprofile eingetragen, in denen sich Hinweise auf eine vorböllingzeitliche klimatische Gunstphase finden. Erfahrungsgemäß sind es besonders die Pollenprofile aus Seeablagerungen, die eine ausreichend mächtige und bei hoher Auflösung untergliederbare präböllingzeitliche Zone enthalten, besonders dann, wenn sie bis in hochglaziale Sedimente zurückreichen. Im fol- genden soll auf einige Pollendiagramme eingegangen werden, deren umfangreiche Spätglazialabschnitte möglicherweise Hinweise auf eine vorböllingzeitliche Gunstphase geben (siehe Abb. 1). Die Vorschläge zu Neueinstufungen wenn stichwortartig, dann in eckigen Klammern eingefügt - stützen sich nur auf palynostratigraphische Argumentation - nicht auf Datierungen.

\section{Poggenwisch (SCHÜTRUMPF 1955):}

Die mächtige Zone I ist untergliederbar und hat bereits an der Basis hohe Artemisia-Werte. Oberhalb der Spektren mit umgelagertem Pollen und hohen Pinus-Werten liegt ein Betula-Gipfel [Meiendorf], auf den unmittelbar ein Hippophaë-Gipfel folgt. Darüber liegt ein deutlicher NBP-Gipfel mit dem absoluten Artemisia- und HippophaëMaximum [Dryas 1]. Erst danach erfolgt die böllingzeitliche Massenausbreitung der Birke.

Usselo (jüngste Bearbeitung van GeEl et al. 1989)

Der als Bölling eingestufte Abschnitt Ib weist niedrigere Birkenwerte als sein Liegendes auf und enthält das Maximum von Hippophaë und Juniperus. Diese Merkmale entsprechen vielmehr der Dyas 1, die Meiendorf von Bölling trennt, als dem Bölling. Im Liegenden befindet sich dann eine Zone (Ia) mit einem langgestreckten Birkengipfel. Dieser könnte als Meiendorf-Interstadial interpretiert werden. Das Bölling-Interstadial würde bei dieser Deutung an der Basis des als Alleröd (II) eingestuften Diagrammbereichs liegen, wo sich ein kurzer, kräftiger Birkenvorstoß befindet.

\section{De Hamert (Teunissen 1983):}

Der Autor setzt das Pollendiagramm in den zeitlichen Rahmen zwischen Bölling und Alleröd. An der Basis des Profils befindet sich die Abfolge: Steiler Betula-Anstieg, Artemisia- und Hippophaë-Maximum [Meiendorf], danach Helianthemum-Maximum und zweiter Artemisia-Gipfel [Dryas 1], dann erst die Massenausbreitung von Betula [Bölling], erneuter NBP-Gipfel [Dryas 2]. Das folgende Alleröd wäre bei dieser Interpretation durch einen NBP-Gipfel unterteilt.

\section{Großes Moor (STREITZ 1984):}

Unterhalb des von der Autorin als böllingzeitlich eingestuften Betula-Gipfels liegt eine Zone erhöhten NBPs (Artemisia!) und ein Strauchpionier-Maximum, an dessen Basis sich das spätglaziale Hippophaë-Maximum befindet [D1]. Darunter zeichnet sich noch ein älterer deutlicher Betula-Vorstoß [Meiendorf] ab. 
Die kritische Sichtung geeigneter Profile aus Süddeutschland liefert auch dort Hinweise auf eine mögliche präböllingzeitliche Gunstphase. So zeigen zum Beispiel im westlichen Alpenvorland die Profile Schleinsee (Mielke \& Müller 1981), Federsee (GöTtLich 1955) und Schussenquelle (LANG 1962) im mächtigen spätglazialen Profilteil einen Birkenvorstoß, der unterhalb des böllingzeitlichen Betula-Maximums liegt. Diese wenigen Beispiele zeigen bereits, daß für das Meiendorf-Interstadial von einer weiterreichenden Gültigkeit als bisher angenommen ausgegangen werden kann.

Aus den Niederlanden liegen zahlreiche spätglaziale Pollendiagramme neueren Datums vor, zuletzt bei Hoek 1997 zusammengetragen. Doch Diagramme mit einem lückenlosen Anschluß an hochglaziale Sedimente bleiben die große Ausnahme. Die Mehrzahl der Pollendiagramme setzt mit dem Bölling-Interstadial oder später ein. Neben dem bereits oben beschriebenen Profil Usselo (van Geel et al. 1989) reicht nur das Profil Mekelermeer (BOHNCKE 1994) noch so weit zurück, daß es die - hier entscheidende - Pollenzone Ia enthält. Das Profil Mekelermeer, die Verfüllung eines Pingos, liegt ca. $70 \mathrm{~km}$ nördlich von Usselo nahe der Vecht. Es enthält zwei deutliche BetulaGipfel, die palynostratigraphisch dem Bölling und Alleröd zugeordnet werden. Die Zone Ia unter dem ersten Betula-Gipfel zeigt insgesamt zwar erhöhte Betula-Werte, jedoch keinen nachfolgenden Rückgang dieser erhöhten Werte, keine ansteigende Artemisia-Kurve und kein HippophaëMaximum, welche gemeinsam die kennzeichnende Merkmalskombination des Meiendorf-Interstadials ausmachen. Daher ist der Einstufung der Zone Ia als Älteste Dryas nichts hinzuzusetzen.

Dieser Profiltyp mit zwei Betula-Gipfeln ist der Grund dafür, daß man in den Niederlanden mit ausschließlich zwei spätglazialen Interstadialen operiert und das Meiendorf als früheste und dritte Wärmeschwankung nicht in Betracht zieht. So erwägen HoEk \& ZAGWIJN (in HoEK 1997: 32-33), die zwei vorhandenen Interstadiale entweder als Bölling-Alleröd oder als Meiendorf-Alleröd zu bezeichnen, letzteres unter Aufgabe des Begriffs Bölling. Bereits Usinger (1985) stellt die Eignung von Bölling Sö als Typlokalität in Frage. Beide von HOEK \& ZAGWIJN angegebenen Lösungen gehen aber von der Voraussetzung aus, daß nur zwei Birkengipfel zuzuordnen sind und würden der Eigencharakteristik des Meiendorf-Interstadials sensu MENKE als biostatigraphische Zone nicht gerecht, denn sie ist eine neu ausgegliederte Zo- ne innerhalb von Ia und ist nicht mit nachfolgenden, jüngeren Birkengipfeln in Ib und II zu verwechseln. Daher wird hier noch einmal betont, daß im Nordwesten Mitteleuropas eine Profilausbildung mit drei palynostratigraphischen sehr typischen und dabei deutlich unterschiedlichen Interstadialen auftreten: Drei Betula-Gipfel liegen in Superposition. Diese drei Birkengipfel müssen zusätzlich in typischer biostratigraphischer Vergesellschaftung vorkommen: Unter einem $\mathrm{Al}$ leröd, das einen oder auch zwei Betula-Gipfel beinhalten kann und minimale NBP-Werte aufweist, folgt eine Zone erhöhter NBP-Werte (D2). Darunter befindet sich ein kurzer markanter Birkenvorstoß (Bölling), der oftmals gleichzeitig das absolute Birkenmaximum des Spätglazials ist. Im Liegenden sind wieder erhöhte NBP-Werte (D1), diesmal gemeinsam mit dem absoluten Maximum der Pioniersträucher, anzutreffen. Besondere Bedeutung haben dabei neben Artemisa vor allem Hippophaë, Juniperus und Helianthemum. Diese Zone wird von einem schwächeren Birkengipfel (Meiendorf) unterlagert, der oft mit ansteigenden Artemisia-Werten zusammenfällt.

Der mittlere kurze und oftmals kräftigste BetulaGipfel wird in allgemeiner Übereinstimmung dem Bölling-Interstadial zugeordnet. Dieses Vorgehen sollte beibehalten werden, unabhängig davon, ob die Typlokalität Bölling Sö als zugehörig bestätigt wird oder nicht. Da das Meiendorf-Interstadial mit seinen typischen Merkmalen stets deutlich unter dem Bölling-Interstadial liegt, sollte eine Verwechslung auszuschließen sein. Damit ist eine Verwendung des Namens Meiendorf als Synonym für Bölling, wie HoeK \& ZAGwIJN (in Hoek 1997: 33) es erörtern, abzulehnen.

Man kann sich natürlich fragen, ob der mitteleuropäische Profiltyp mit den drei Interstadialen Meiendorf-Bölling-Alleröd in den Niederlanden eine Westgrenze erfährt. Solange aber in den Niederlanden nicht mehr Profile der hier dargestellten Ausbildung und Vollständigkeit gefunden werden, läßt sich über eine Abgrenzung dieses Profiltyps keine Entscheidung fällen.

Abschließend soll auf Veröffentlichungen hingewiesen werden, die sich mit frühen spätglazialen Erwärmungsphasen außerhalb von Mitteleuropa befassen.

\section{Vorböllingzeitliche Wärme- schwankungen außerhalb Mitteleuropas}

Vorböllingzeitliche Wärmeschwankungen außerhalb Mitteleuropas sind zahlreich dokumentiert. Seit 1915 LEVERETT \& TAYLOR im Gebiet der Großen 
Seen Nordamerikas die Moränen der WisconsinVereisung und deren Spätglazial kartiert haben, gibt es aus dem Bereich des proglazialen Sees Arkona erste quartärgeologische Belege für eine Wärmeschwankung, die jünger als das letztglaziale Maximum (LGM) und älter als das Bölling ist.

Sie erhielt den Namen Lake Arkona-Schwankung. Seit den 50er Jahren häuften sich palynologische Hinweise auf möglicherweise vergleichbare vorböllingzeitliche Interstadiale sowohl auf der Nordhalbkugel wie auch auf der Südhalbkugel. Palynologische Belege zu den zahlreichen glazialgeologischen Befunden aus dem Bereich der Großen Seen lieferten zum Beispiel aus Connecticut das Pollenprofil Totoket Bog (Leopold 1956), aus Ohio das Profil von Torrens Bog (Ogden \& Hay 1965). Dadurch konnte das Ende des Lake Arkona-Interstadials mit ${ }^{14} \mathrm{C}$-Daten auf den Zeitraum um 13.000 a BP festgelegt werden. In Kolumbien wiesen VAN DER HAMMEN \& GONZALES (1965) die Susacá-Wärmeschwankung nach, die VAN DER HAMMEN \& VOGEL (1966) mit Befunden aus Kenia, Frankreich und Spanien korrelierten. Die Radiokarbondaten für die Abgrenzung des Susacá-Interstadials liegen bei 13.700-13.100 a BP. MÖRner (1970) parallelisiert das Susacá- und das Lake Arkona-Interstadial mit dem Raunis-Interstadial (SEREBrjannYI \& RauKas 1970) im östlichen Baltikum. ${ }^{14} \mathrm{C}$-datierte Pflanzenlagen ergaben für letzteres zwei Daten: $13.890 \pm 500$ und $13.250 \pm$ $160 \mathrm{a}$ BP.

Die vorgetragenen Daten gruppieren sich allesamt zwischen 14.000 und 13.000 a BP. Unter Einbeziehung der Fehlergrenzen könnten sie theoretisch ein und dasselbe Interstadial bezeichnen. Für die Untergrenze des Meiendorf-Interstadials gibt Menke (in Druckvorbereitung) das ${ }^{14} \mathrm{C}$-Alter $12.660 \pm 300$ a BP und für die Obergrenze 12.010 \pm 75 a BP an. Das Alter für den Beginn des Meiendorf-Interstadials entnimmt er dem ArtemisiaAnstieg im Profil Usselo (van GeEl et al. 1989) und dasjenige für das Ende dem NBP-Anstieg nach dem Hippophaё-Maximum im Profil Esinger Moor (Bokelmann et al. 1983). Wenn auch das Alter für die Obergrenze Meiendorf zu jung erscheint denn es blieben für Dryas 1, Bölling und Dryas 2 nur etwa 200 Radiokarbonjahre - so ist dennoch das Meiendorf-Interstadial nach ${ }^{14} \mathrm{C}$-Alter deutlich jünger als die oben diskutierten Interstadiale außerhalb Mitteleuropas. In Glazialgebieten können diese älteren schwimmenden Interstadiale nur jeweils zwischen die äußerste Eisrandlage des Oberwürms und das Bölling-Interstadial eingeengt werden. Ansonsten ist ihre stratigraphische
Stellung nur durch Radiokarbondatierungen festgelegt.

Es zeigt sich jedoch ferner, daß für den Zeitraum zwischen dem LGM und Bölling eine ganze Reihe von leichten Wärmeschwankungen nachgewiesen sind. Mania \& STECHEMESSER (1970) beschreiben auf der Basis von Molluskenfaunen eine zweigeteilte Wärmeschwankung aus dem Geiseltal als Müchelner Intervall und plazieren es vor das Bölling-Interstadial. MANIKOWSKA (1995 a, b) beschreibt aus Zentralpolen die Klimaverbesserung der Kamion-Phase mit einer Bodenbildung und ${ }^{14} \mathrm{C}$-Daten von $14.300 \pm 300$ und $14.590 \pm 270$ a BP. Kolstrup (1980) berichtet aus den Niederlanden von der organischen Lage des Epe-Horizonts mit einem Alter von $14.000 \pm 150$ a BP. W. SCHIRMER (im Druck) berichtet vom Leonard-Boden, einer Kalkbraunerde im jüngsten Rheinlöß, die als Indiz für eine Wärmeschwankung nach dem LGM und vor dem Beginn des Spätglazials gilt.

Schon diese wenigen bodenkundlichen, malakologischen und glazialgeologischen Beispiele zeigen anschaulich, daß seit dem Rückzug des Eises vom LGM bis zum Spätglazial offenbar eine höhere Anzahl kleinerer Wärmeschwankungen zumal von der geringen Größenordnung des MeiendorfInterstadials zu erwarten ist. Solange solche Schwankungen zwischen dem LGM und dem Bölling isoliert auftreten, sei es ohne Datierung oder nur durch ${ }^{14} \mathrm{C}$-Alter belegt, ist ihre Korrelation untereinander und mit dem MeiendorfInterstadial vorerst wenig sinnvoll, wenn sie nicht mit dem bekannten Spätglazial in direktem Profilkontakt stehen.

Ich danke dem anonymen Rezensent für seine Anregungen.

\section{Schriftenverzeichnis}

Aletsee, L. (1959): Zur Geschichte der Moore und Wälder des nördlichen Holsteins. - Nova Acta Leopoldina, N.F. 21 (139): 51 S., 9 Taf. als Beil; Leipzig.

Bock, W., Menke, B., Strehl, E. \& Ziemus, H. (1985): Neuere Funde des Weichselspätglazials in Schleswig-Holstein. - Eiszeitalter und Gegenwart, 35: 161180; Hannover.

BoHncke, S. J. P. (1994): Lateglacial environmental changes in The Netherlands: Spatial and temporal patterns. - Quaternary Science Rewievs, 12: 707717; London.

Bokelmann, K., Heinrich, D. \& Meyer, B. (1983): Fundplätze des Spätglazials am Hainholz-Esinger Moor, Kreis Pinneberg. - Offa, 40: 199-239; Neumünster. 
Dreimanis, A. (1966): The Susacá-Interstadial and the subdivision of the Late-Glacial. Discussion. - Geologie en Mijnbouw, 45: 445-448; 's-Gravenhage.

FIRBAS, F. (1949): Spät- und nacheiszeitliche Waldgeschichte Mitteleuropas nördlich der Alpen. - 480 S.; Jena

Geel, B. van, Coope, G. R. \& Hammen, T. van der (1989): Palaeoecology and stratigraphy of the Lateglacial type section at Usselo (The Netherlands). - Rev. Palaeobot. Palynol., 60: 25-129; Amsterdam.

GöTtLICH, K. (1955): Ein Pollendiagramm ungestörter späteiszeitlicher Verlandungsschichten im Federseebecken. - Beitr. z. naturkundl. Forsch. in Südwestdeutschland, 14: 88-92; Karlsruhe.

Hammen, T. van der (1951): Late-Glacial flora and periglacial phenomena in The Netherlands. - Leidse Geol. Mededelingen, 17: 71-184; Leiden.

- \& Gonzales, E. (1965): A Lateglacial and Holocene pollen diagram from Cienaga del Visitador (Dept. Boyaca, Colombia). - Leidse Geol. Meded., 32: 193201, Leiden

— \& Vogel, J. C. (1966): The Susacá-Interstadial and the subdivision of the Late-Glacial. - Geologie en Mijnbouw, 45: 33-35; s'-Gravenhage.

Hartz, N. \& Milthers, V. (1901): Det senglaciale Ler i Alleröd Teglvaerkgrav. - Medd. Danm. geol. Foren., 8: 31-60; Kopenhagen.

HoEk, W. (1997): Palaeogeography of Lateglacial Vegetations. - Nederlandse Geografische Studies, 230: 147 S.; Utrecht, Amsterdam.

Iversen, J. (1942): En pollenanalytisk Tidfaestelse af Ferskvandslagene ved Nörre Lyngby. - Medd. Danm. geol. Foren., 10 (2): 130-151; Kopenhagen.

Kolstrup, E. (1980): Climate and stratigraphy in northwestern Europe between 30,000 B.P. and 13,000 B.P. with special reference to the Netherlands. - Meded. Rijks Geol. Dienst, 32: 181-253; Haarlem.

LANG, G. (1962): Vegetationsgeschichtliche Untersuchungen der Magdalenienstation an der Schussenquelle. - Veröff. d. Geobotanischen Instituts der ETH Zürich, 37: 129-154; Bern.

Leopold, E. B. (1956): Two late-glacial deposits in southern Connecticut. - Nat. Acad. Sci. Proc., 42: 863-867; Washington D.C.

Leverett, F. \& TAylor, F. B. (1915): The Pleistocene of Indiana and Michigan and the history of the Great Lakes. - U. S. Geol. Survey Monogr., 53: 529 p.; Washington D.C.

LitT, T. \& Stebich, M. (1996): Annually laminated sediments from Holzmaar (Weichselian Late-Glacial). 10. Moorexkursion 10.-19. Oktober 1996: 80-84; Geobotanik Univ. Bern.

Mania, D. \& Stechemesser, H. (1970): Jungpleistozäne Klimazyklen im Harzvorland. - Petermanns geogr. Mitt., Ergänzungsband 274: 39-55; Gotha, Leipzig.

MANikowskA, B. (1995 a): The apogee of Vistulian cold in the periglacial area of Central Poland - geological records. - Quaternary Studies in Poland, 13: 55-63; Warszawa.
- (1995 b): Aeolian activity differentiation in the area of Poland during the period 20-8 ka BP. - Biuletyn Peryglacjalny, 34: 127-165, Tab. 1; tódź.

Menke, B. (1968): Das Spätglazial von Glüsing. - Eiszeitalter und Gegenwart, 19: 73-84; Öhringen.

- Jungpleistozäne Biostratotypen in Norddeutschland. - Geol. Jb. (in Druckvorbereitung).

Merkt, J. \& Müller, H. (1999): Warve chronology and palynology of the Late Glacial in Northwest Germany from lacustrine sediments of Hämelsee in Lower Saxony. - Quaternary International, in print.

Mielke, K. \& Müller, H. (1981): Palynologie. - In: BeNDER, F. [Hrsg.]: Angewandte Geowissenschaften, 1: 393-407, Abb. 6.9; Stuttgart (Enke).

Mörner, N.-A. (1970): Comparison between Late Weichselian and Late Wisconsin ice marginal changes. - Eiszeitalter und Gegenwart, 21: 173-176; Öhringen/Württ.

NiLsson, T. (1961): Ein neues Standardpollendiagramm aus Bjärsjöholmssjön in Schonen. - Lunds Universitets Arksskrift, N. F. Avd. 2, 56 (18) (Kungl. Fysiografiska Sällskapets Handlingar, NF. 71`(18): 1-34; Lund.

Ogden, J. G. III \& Hay, R. J. (1965): Ohio Wesleyan University natural radiocarbon measurements I. - Radiocarbon, 7: 166-173; Tucson.

SCHIRMER, U. (1995): Early Late Glacial pollen record of Miesenheim. - In: Schirmer, W. [ed.]: Quaternary field trips in Central Europe, 1: 533-535; München (Pfeil).

- (1996): Pollen stratigraphy below the Pellenz tephra. - In: Schirmer, W., Ikinger, A., Schiermeyer, J., Schirmer, U. \& Waldmann, G. (1996): Guide to the archives of the Laacher See eruptions: 15-17; Düsseldorf (Dept. Geol. HHUniversity).

- (1998): Spätglaziale Vegetationsgeschichte an der Lahn. - GeoArchaeoRhein, 2: 163-175; Münster (Lit).

Schirmer, W. (im Druck): Eine Klimakurve des Oberpleistozäns aus dem rheinischen Löß. - Eiszeitalter und Gegenwart.

SchütrumpF, R. (1936): Paläobotanisch-pollenanalytische Untersuchung der paläolithischen Rentierjägerfundstelle von Meiendorf bei Hamburg. - Veröff. d. Archäol. Reichsinst., 1: 1-54; Neumünster.

- (1955): Das Spätglazial. - Eiszeitalter und Gegenwart, 6: 41-51; Öhringen.

SerebrjannyJ, L. R. \& Raukas, A. V. (1970): Über die eiszeitliche Geschichte der Russischen Ebene im oberen Pleistozän. - Petermanns geogr. Mitt., 144 (3): 161-172; Gotha, Leipzig.

Streitz, B. (1984): Vegetationsgeschichtliche Untersuchungen an zwei Mooren osthessischer Subrosionssenken. - Beitr. Naturkde. Osthessen, 20: 3-77; Fulda.

Teunissen, D. (1983): The development of the landscape of the nature reserve De Hamert and its environs in the northern part of the province of Limburg, The Netherlands. - In: Terwindt, J. H. J. \& 
STEIJN, H. VAN [eds.]: Developments in physical geography - a tribute to J. I. S. ZonnevelD. - Geol. Mijnbouw, 62: 569-576; 's-Gravenhage.

Usinger, H. (1985): Pollenstratigraphische, vegetationsund klimageschichtliche Gliederung des „BöllingAlleröd-Komplexes" in Schleswig-Holstein und die Bedeutung für die Spätglazial-Stratigraphie in benachbarten Gebieten. - Flora, 177: 1-43; Jena.

Manuskript eingegangen am 5. März 1999 